Check for updates

Cite this: Mater. Adv., 2020,

1,2483

Received 10th July 2020,

Accepted 20th September 2020

DOI: $10.1039 / \mathrm{d} 0 \mathrm{ma00495b}$

rsc.li/materials-advances

\title{
Gliadin-coated gold nanoparticles for rapid colorimetric test for celiac disease $\dagger$
}

\author{
Anantdeep Kaur, ${ }^{a b}$ Ying Wang, ${ }^{a}$ Michael Wallach* ${ }^{\mathrm{b}}$ and Olga Shimoni (D) *ac
}

\begin{abstract}
Celiac disease (CD) is an immune-mediated disorder affecting the small intestine in genetically predisposed individuals. Despite the significant progress made in research on CD, there is still an urgent need for accurate detection or a point-of-care test for the rapid diagnosis of the disease. In this paper, we present a novel detection test for CD based on the coating of gold nanoparticles with gliadin, the highly antigenic protein that induces CD. Although the protein is cheap and stable, its hydrophobic nature prevented its efficient use in diagnostics. In our work, we successfully demonstrated the binding of the whole hydrophobic gliadin protein to the surface of the gold nanoparticles that we further translated into a simple assay for the detection of CD specific biomarkers in serum as well as saliva. Finally, we compared the diagnostic accuracy of the novel test on 30 previously tested patient serum samples and saliva from 35 untested participants in a blinded assessment that were further compared to the previous serological and pathological tests on those patients. The data showed that the developed test using gold nanoparticles had an overall accuracy of over $96 \%$ in detecting celiac disease. Our developed test method offers a substantial advantage over the existing blood tests by eliminating multiple steps associated with routine serological testing, providing results within 15-30 minutes that can be determined by eye without any specialized equipment for signal reading. Finally, the developed assay is valuable for testing CD patients using saliva as a non-invasive, point of care test.
\end{abstract}

\section{Introduction}

The field of diagnostics has experienced explosive growth over the last decade. ${ }^{1,2}$ Portable diagnostic kits are highly sought after as they offer a relatively fast, easy and simple technology to detect a large number of biomarkers. Despite these advancements, there are still diseases that would greatly benefit from developing a lab-based or point-of-care diagnostic test. One such condition is celiac disease.

Celiac disease (CD) is a chronic illness that affects the small intestine due to an auto-immune reaction induced by the consumption of gluten, a protein found in wheat and other cereal grains. ${ }^{3}$ Epidemiological studies have shown that there is a worldwide distribution of celiac disease with a high proportion of CD sufferers going undiagnosed. ${ }^{4}$ Early diagnosis is vital

\footnotetext{
${ }^{a}$ Institute for Biomedical Materials and Devices, Faculty of Science, The University of Technology Sydney, 15 Broadway, Ultimo, Sydney, New South Wales, 2007, Australia. E-mail: olga.shimoni@uts.edu.au

${ }^{b}$ School of Life Sciences, Faculty of Science, The University of Technology Sydney, 15 Broadway, Ultimo, Sydney, New South Wales, 2007, Australia ${ }^{C}$ ARC Research Hub for Integrated Device for End-user Analysis at Low-levels (ARC IDEAL Hub), Faculty of Science, University of Technology Sydney, 15 Broadway, Ultimo, NSW 2007, Australia

$\dagger$ Electronic supplementary information (ESI) available. See DOI: 10.1039/ d0ma00495b
}

in order to prevent the progression of $\mathrm{CD}$ that can often lead to severe complications.

Symptoms of CD vary significantly in different individuals and include anemia, anorexia, weight loss, abdominal pain, diarrhea, chronic fatigue, constipation, joint pain as well as an increased level of liver enzymes. ${ }^{5}$ Due to the wide variability in symptoms and similarity to other gastrointestinal disorders, such as Crohn's disease and Inflammatory Bowel Disease (IBD), it has proven difficult to correctly diagnose $\mathrm{CD}$ at an early stage by a medical practitioner.

The first step for CD diagnosis in clinical practice is serologic testing for gliadin-induced autoantibodies; the two key ones are an anti-gliadin antibody (AGA) and a tissue antitransglutaminase (tTG) antibody. The most reliable method for diagnosing CD, however, is gastrointestinal endoscopy, which is invasive, costly, and time-consuming. This method relies on the clinician to acquire a tissue sample for a mucosal biopsy for visualization of varying degrees of intestinal damage, from mild abnormalities to completely flat mucosa. ${ }^{3,6}$ In addition, genetic testing for individuals with a high probability of developing $\mathrm{CD}$, i.e., those showing the presence of the main susceptibility genes HLA-DQ2 (specifically HLA-DQ2.5 and HLA-DQ2.2) and HLA-DQ8, is also used in combination with the other serological approaches for diagnosis. ${ }^{7}$ Typically, CD patients present higher serum titers of AGA and anti-tTG 
antibodies that are detectable using a lab-based, enzyme-linked immunosorbent assay (ELISA) test. ${ }^{8,9}$ In non-CD patients, these two types of antibodies are generally undetectable in serum.

Several commercial kits based on the serological testing of antibodies against tTG and DGP have been introduced and used for large-scale screening of the general population. However, the existing point-of-care methods for identifying CD are unable to provide the required diagnostic accuracy that might be affected by the higher variability in the characteristics of patients, such as their age, family history, or other clinical conditions associated with auto-immune diseases. ${ }^{10}$

Over the last decade, nanoparticle-based technology has been applied to the early detection of multiple diseases, such as HIV ${ }^{11,12}$ other pathogenic infections, ${ }^{13,14}$ pregnancy testing in women, ${ }^{15}$ and many other applications. ${ }^{16-20}$ This technology has proven to be highly sensitive, accurate, and easy to perform. Nanotechnology-based approaches have been employed to deliver a point-of-care test for $\mathrm{CD}$ detection, involving the coating of DGP on a carrier protein as the antigen and using colloidal gold anti-human antibodies as the signal detector to identify anti-DGP antibodies in serum samples. Although it is a plausible approach, the test was found to be less specific than expected, which limited its potential as a population screening tool due to the functional complexity of the method. ${ }^{21}$

In this paper, we demonstrate a simple, cheap and accurate colorimetric immunoassay to detect celiac disease-specific anti-gliadin antibodies in bodily fluids. To exhibit it, (1) we assembled a stable suspension (without any significant level of aggregation) of gold nanoparticles (AuNPs) coated with the hydrophobic whole gliadin protein; (2) we adapted and validated it to develop a novel screening test for rapid detection of celiac disease using simulated bodily fluids (Fig. 1). Interestingly, we found that the use of the whole gliadin protein with AuNPs showed a better binding towards antibodies than in

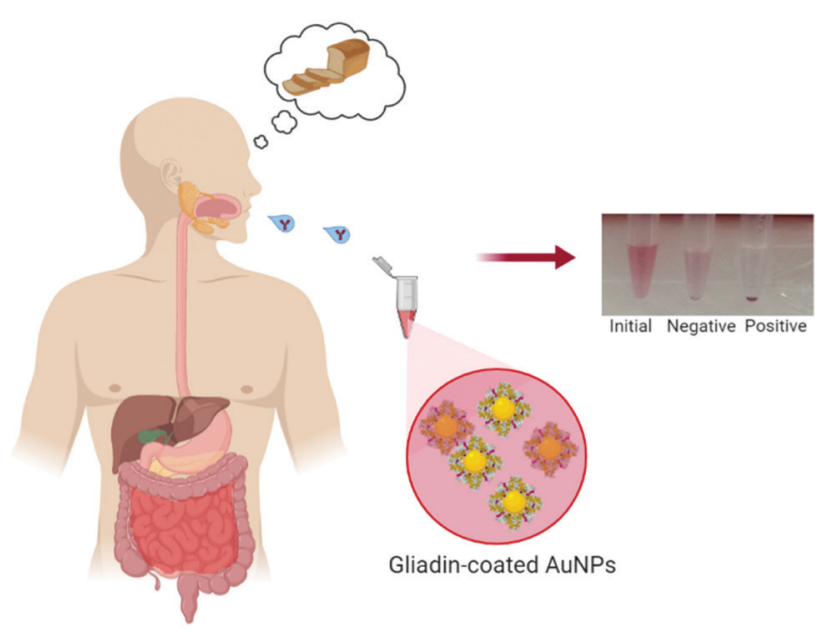

Fig. 1 Rapid screening test for celiac disease. Schematic representation of the gliadin-coated immunoassay for fast and accurate detection of the celiac disease biomarker in saliva. The colorimetric output can be observed by a naked eye, where AGA binding to gliadin-coated AuNPs induces nanoparticle aggregation, leading to a change to a change in color. The absence of the AGA causes just a bit of color weakening. typical flat surface tests (i.e., ELISA), and we predict that the curvature of AuNPs' increased the exposure of hidden epitopes in gliadin towards antibody recognition. (3) Importantly, we tested our invention on 30 patient serum and 35 saliva samples and found that the assay achieved a high level of accuracy $(>95 \%)$ in distinguishing CD from non-CD patients. We contemplate that this immunoassay represents a simple, straightforward nanoparticle-based assay for the detection of celiac disease that can be easily translated into clinical practice.

This level of accuracy is in the range required for a serological testing while in the clinic or home we would expect to find only a relatively small proportion of people tested with CD. In addition, these results for the detection of AGA eliminate multiple steps followed in existing serological tests that sometimes lead to user-dependent error. A test enabling early detection would be particularly useful in aiding the large-scale screening of the general population, particularly in the preselection of CD in small children by parents or medical practitioners, which can be then be confirmed by mucosal biopsy.

\section{Results and discussion}

Gliadin protein, found in wheat, barley, and rye, is the antigenic protein that induces CD in genetically predisposed people. Gliadin epitopes are recognized by $\mathrm{T}$ cells that stimulate the formation of CD4, natural killer (NK)-like cells, as well as the release of pro-inflammatory cytokines such as IFN- $\gamma$ thereby, activating the adaptive immune response. ${ }^{22,23}$ Typically, patients with CD present higher serum titers of AGA and anti-tTG antibodies that are detectable using a lab-based, enzyme-linked immunosorbent assay (ELISA) tests. ${ }^{8,9}$

Over the years, tTG antibodies showed higher specificity and sensitivity values as compared to AGA tests, which led to the abandoning of whole gliadin protein-specific AGA as the biomarker for diagnosing CD. More recently, ELISA tests using human recombinant tTG (h-tTG) or deamidated gliadin peptide (DGP) as the antigen source have further improved the sensitivity of CD using the patient's blood serum. However, increased tTG antibody titers are also associated with Type I diabetes as well as some liver disorders, and this can generate an unacceptable number of false-positive results.

One of the reasons that AGA lacks high specificity towards gliadin is that gliadin is a mostly hydrophobic wheat protein that is only slightly soluble in aqueous solution. It is challenging to use this protein as an antigen in typical ELISA testing.

To use gliadin as an antigen and to coat AuNPs for AGA detection, we overcame the problem of aggregation of the coated nanoparticles. In previous studies using coated AuNPs, the vast majority of work used water-soluble molecules, such as DNA/RNA, ${ }^{24-26}$ peptides, ${ }^{27,28}$ aptamers $^{29}$ and albumins, ${ }^{30}$ to coat the nanoparticles. Because of the hydrophobicity of the used protein, we had to develop a new method to achieve a stable colloidal suspension of gliadin-coated AuNPs under physiological conditions. 
We tested a variety of protic and aprotic solvents, such as acetone, chloroform, dichloromethane, dimethylformamide and methanol, where we observed only partial solubility of the protein. We developed a protocol for sufficient solubilization of gliadin using the cationic surfactant CTAB and $70 \%$ isopropanol previously used in other studies to extract some plant-based proteins. ${ }^{31}$ In the aqueous environment, gliadin would have a compact globular shape with polar groups and hydrophilic amino acids oriented outwards as it is considered energetically favourable conformation. Gliadin protein has a considerate amount of glutamine amino acids in its sequence, ${ }^{32}$ and they can interact with the gold surface due to high affinity for amines, thiols and phosphines. ${ }^{34}$ The use of CTAB surfactant provides a further stabilization of the nanoparticles by incorporation steric hindrance and positive charge leading to electrostatic repulsion between nanoparticles.

In the present study, $20 \mathrm{~nm}$ citrate stabilized gold nanoparticles were used. The citrate layer provides long term stability to the AuNPs and is weakly associated with the nanoparticle surface. This citrate layer can be easily displaced by a range of molecules, including proteins. Adsorption of gliadin on the surface of AuNPs followed by displacement of the citrate layer involved non-covalent processes based on the ionic interactions between the negatively charged nanoparticle and the positively charged amino acids in the protein. Some interactions also occurred due to covalent binding between the AuNPs and the conducting electrons of nitrogen and sulphur atoms present in the protein. ${ }^{33}$ These interactions help in providing stability to the coated AuNPs in the aqueous solution.

The solubilization of gliadin allowed us to adsorb the protein on the surface of AuNPs, which we monitored using UV-Vis spectrophotometry measurements. ${ }^{34}$ We found that there was a redshift in the absorbance maximum from $525 \mathrm{~nm}$ to $532 \mathrm{~nm}$, indicating a shift in the localized plasmon resonance of AuNPs due to the protein adsorbed onto the AuNPs surface (Fig. 2A). In addition, we did not observe a measurable decrease of absorbance at peak wavelength or an increase of absorbance at longer wavelengths (600-700 $\mathrm{nm})$, suggesting the colloidal dispersion of protein-adsorbed AuNPs was stable, and no strong AuNP-toAuNP interactions or aggregation occurred.

We further confirmed there was no aggregation using DLS, which showed an increase in hydrodynamic diameter from $20 \mathrm{~nm}$ to $28 \mathrm{~nm}$ following adsorption with gliadin (Fig. 2B). As the molar mass of the protein is directly proportional to its hydrodynamic radius in solution, ${ }^{35}$ an increase in the hydrodynamic radius indicates that the coating of AuNPs with gliadin had occurred. We carried out a similar test using control AuNPs absorbed with BSA (molecular weight $=66 \mathrm{kDa}$ ) protein, where we found an average hydrodynamic particle diameter of $32 \mathrm{~nm}$, correlating with the larger size of the protein.

We observed zeta potential change of AuNPs as a result of gliadin absorption. The value changed from $-27.5 \mathrm{mV}$ for citrate-capped AuNPs to $+18.1 \mathrm{mV}$ after absorption of gliadin. This further confirms the assumption that in the aqueous solution gliadin protein present polar amino acids (mostly glutamine) on the surface.
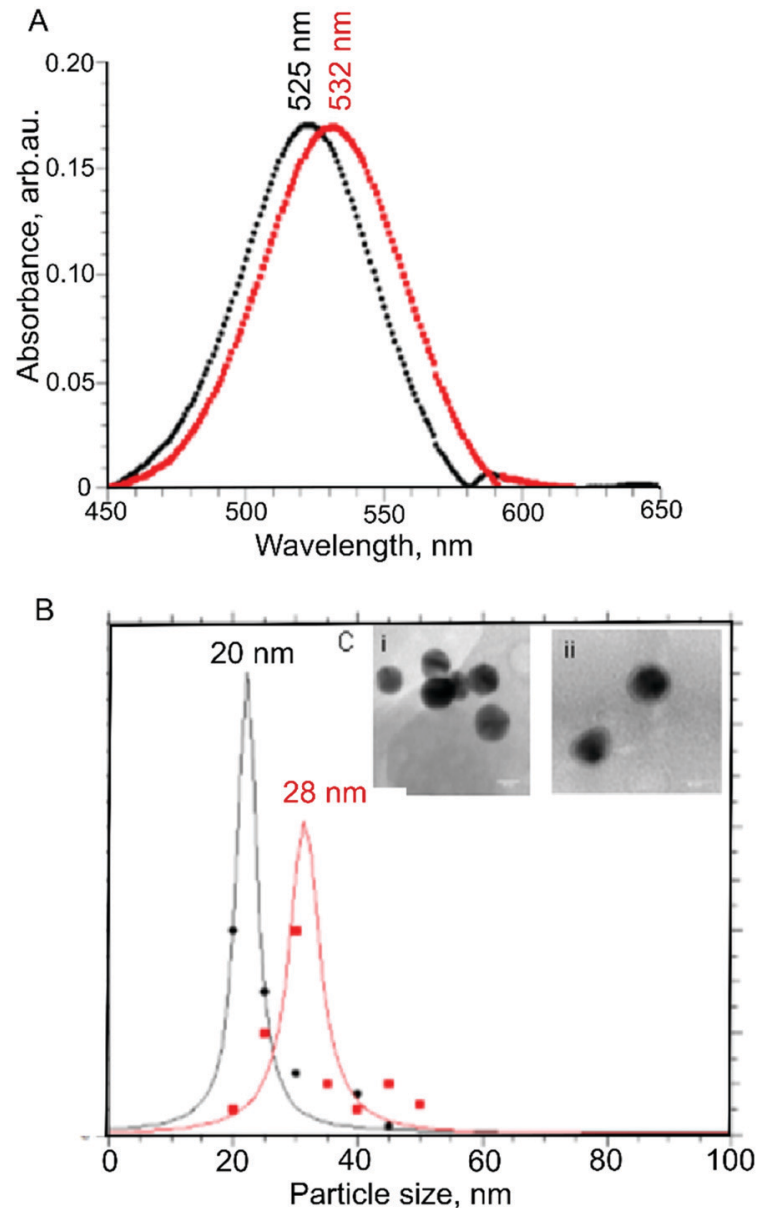

Fig. 2 Characterization of gliadin coated AuNPs. (A) Characterization of AuNPs adsorbed with gliadin using a UV-Vis Spectrophotometer show a spectral redshift in wavelength from $525 \mathrm{~nm}$ (for $20 \mathrm{~nm}$ AuNPs only) to $532 \mathrm{~nm}$ (for $20 \mathrm{~nm}$ AuNPs adsorbed with gliadin). (B) Characterization of AuNPs adsorbed with gliadin using DLS, showing an increase in the hydrodynamic size of the uncoated vs. coated particles from $20 \mathrm{~nm}$ to $28 \mathrm{~nm}$, respectively. (C) High-resolution TEM images of (i) uncoated AuNPs, (ii) gliadin coated AuNPs showing a 'halo' layer indicating coating of the gold with the protein had occurred. In contrast, the 'halo' effect was not observed on the surface of the un-coated AuNPs.

To directly observe gliadin absorbed on AuNPs, highresolution TEM imaging was used. The presence of a thin layer of material (1-2 nm) (Fig. 2C(ii)) surrounding the nanoparticles, which was not observed on the surface of the uncoated nanoparticles (seen in Fig. 2C(i)) further confirmed the absorption of protein. ${ }^{36}$

\section{Immunoassay of gliadin-coated AuNPs with AGA}

To examine the ability of gliadin-coated AuNPs to detect AGA, we first tested serial dilutions of rabbit anti-gliadin IgG polyclonal antibody (AGA) in a range that exists typically in human serum. ${ }^{37,38}$ After 30 minutes incubation, we observed a significant reduction in color as well as a decrease in the absorbance peak with a shift from $532 \mathrm{~nm}$ to $580 \mathrm{~nm}$ (Fig. 3A). These results showed that in the presence of the AGA, increased nanoparticleto-nanoparticle interaction leads to aggregation and precipitation 
A
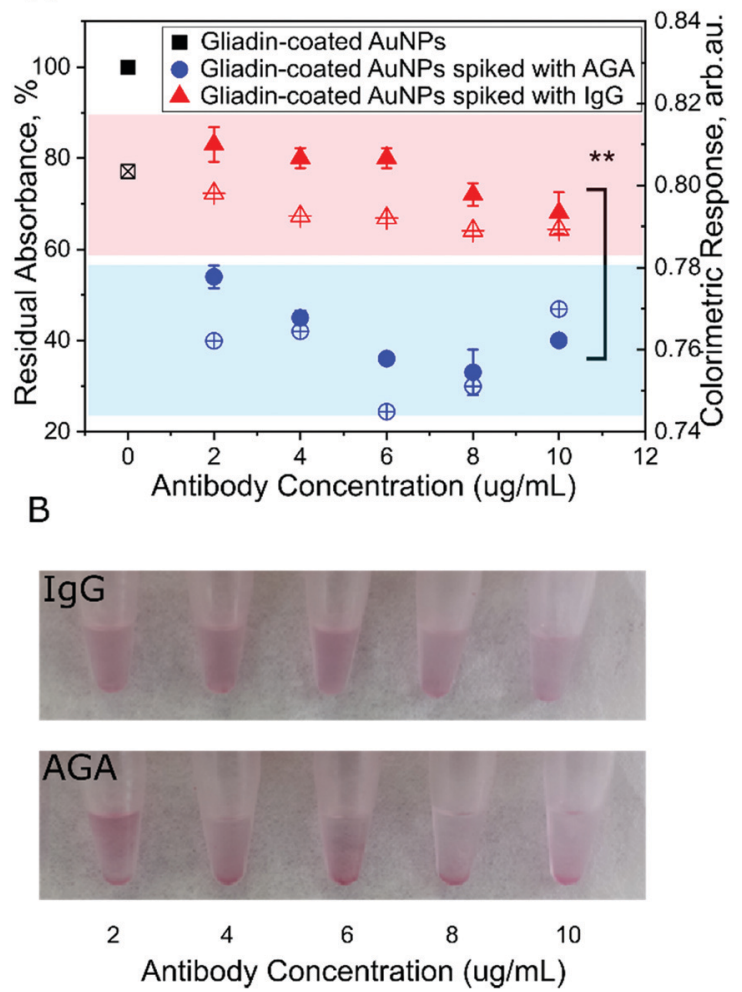

Fig. 3 Testing gliadin-coated AuNPs in buffer with spiked AGA (positive test) or IgG from human serum(control). (A) Change in absorbance (filled markers) and colorimetric response (open markers) after incubation with different concentrations of antibodies (Blue markers for AGA and red markers for control lgG antibodies). The initial solution of AuNPs coated with gliadin in water was defined as $100 \%$ absorbance. (B) Visual performance: no significant change in color or shift in peak wavelength was observed in gliadin coated AuNPs incubated with control lgG (top)at all dilutions tested $\left(2,4,6,8,10 \mu \mathrm{g} \mathrm{mL}^{-1}\right)$ while there is visible precipitation formed in samples after mixing with AGA (bottom).

of the AuNPs. Interestingly, we have not observed color change to blue/purple. While the AuNPs absorb and scatter light intensely at their surface plasmon resonance (SPR) frequency that is sensitive to the inter-particle distance between the nanoparticles. The color change happens due to aggregation of AuNPs that increases electric dipole-dipole interaction and overlapping of plasmons of neighbouring particles. However, in this study when the interparticle distance is substantially greater than the average particle diameter, the AuNPs appear red. Specifically, if we take into account the size of the protein adsorbed on the surface of the AuNPs (4-5 nm) together with the complex of antibody ( $10 \mathrm{~nm})$, it creates a greater distance between adjacent nanoparticles. Therefore, the aggregates formed in this study precipitate out and appear as red while leaving the suspension translucent.

As a control, we tested normal serum IgG antibody with the gliadin-coated AuNPs. We found that there was no significant change in color or shift in the wavelength or size of the absorbance peak after taking the dilution factor into account (Fig. 3A and B). We found that at all the tested concentrations, the absorbance was significantly lower using AGA as compared to normal IgG, reaching its minimum value in the range of
4-8 $\mu \mathrm{g} \mathrm{mL} \mathrm{m}^{-1}(p<0.005$, Fig. 3A). These results demonstrate the specificity and sensitivity of the assay.

We tested uncoated AuNPs and those coated with an irrelevant antigen, such as BSA, incubated with AGA as controls for specificity. Our results did not show any significant change in absorbance or aggregation (Fig. S3 and S4, ESI $\dagger$ ), confirming the specificity of the assay for whole gliadin.

To assess the specificity of the AGA toward gliadin coated AuNPs, we calculated a colorimetric response using our assay (Fig. 2C and Fig. S5, ESI $\dagger$ ). We found that the response reaches a maximum value at a concentration of $6 \mu \mathrm{g} \mathrm{mL} \mathrm{L}^{-1}$ of AGA. The near-constant colorimetric response curve obtained for the control antibody as compared to the response curve obtained for AGA further demonstrates the specificity of the assay.

\section{Testing AGA in spiked serum and saliva}

Human serum is a complex fluid containing various proteins, peptides, exosomes as well as nucleic acids. To reduce background binding, we used $1 \%$ BSA as a blocking agent to lower the non-specific interaction with the gliadin-coated AuNPs. Spiked human serum containing $2-10 \mu \mathrm{g} \mathrm{mL}^{-1}$ AGA was incubated with gliadin-coated AuNPs. We observed a reduction in the color of the solution from red to translucent coupled with the formation of aggregation and precipitation, which was easily detectable by eye (Fig. 4A). This change was further supported by UV-Vis measurements, where the maximum change was observed at $8 \mu \mathrm{g} \mathrm{mL} \mathrm{m}^{-1}$ of AGA (Fig. S6 and S7, ESI $\dagger$ ). In contrast, when we added normal IgG to the human serum, no precipitate formation or change in color was observed. The normal serum itself did not show any change

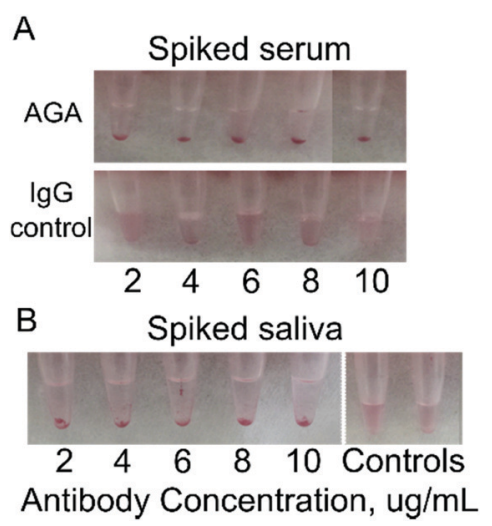

Fig. 4 Detection of AGA in spiked human serum and saliva samples using gliadin-coated AuNPs. (A) AuNPs adsorbed with gliadin showed intense precipitation in the presence of AGA and serum at different concentration. The precipitation can be observed by the naked eye within 20 min after incubation. No reduction in color from red to translucent or precipitate formation was observed in AuNPs adsorbed with gliadin in the presence of control lgG and serum. All serum samples were diluted 1:20 before the addition of antibodies. (B). Testing gliadin-coated AuNPs with AGA in saliva at different dilutions. Reduction in color from red to translucent and formation of precipitation was clearly observed in AuNPs coated with gliadin incubated with AGA at various dilutions. As human saliva contains multiple antibodies, no spiking of control antibodies was required. Saliva samples were only slightly diluted. 
in absorbance, and the solutions preserved a pink color over a long period of time (Fig. 4A).

Human saliva is another complex fluid that contains multiple molecular species. Developing a test that would be able to detect biomarkers in the saliva is of utmost interest for truly non-invasive detection. In this work, we first tested the ability of gliadin-coated AuNPs to detect AGA in spiked saliva from healthy volunteers. The antibody concentrations used in saliva were similar to those typically observed in the saliva of patients with $\mathrm{CD}^{39,40}$

Human saliva spiked at various concentrations of AGA was mixed with gliadin coated AuNPs, and the results we observed after $20 \mathrm{~min}$ by the naked eye. We confirmed that the gliadinAuNPs were able to interact with AGA in saliva samples (Fig. 4B). AGA-spiked saliva showed a visible precipitate while control samples remained pink in color. Human saliva did not require spiking with control antibodies as it already contains large amounts of IgG and IgA endogenous antibodies, peptides, nucleic acids and more. When observed by UV-Vis spectrometry, a decrease in the absorption wavelength was detected when the gliadin-coated AuNPs were added to saliva samples spiked with AGA. Normal saliva itself did not show any absorbance change. These readings confirmed that the AGA could trigger aggregation in saliva, and their specificity is not affected by other constituents in saliva. A change in color from red to translucent was clearly observed (Fig. 4B).

\section{Clinical samples testing}

To assess the clinical relevance of the developed method, we next used the gliadin-coated AuNPs to test a selected set of human serum and saliva samples obtained from patients with CD or healthy controls. The goal of the small clinical study was to evaluate the ability of the assay to distinguish between previously diagnosed CD patients from non-CD individuals. The testing of clinical samples has been performed blinded without prior knowledge of the clinical outcome for the tested patient's serum and saliva.

The results for the 30 clinical serum samples and 35 saliva samples were recorded after the visual examination of precipitate formation and the determination of a shift or change in absorbance values using a UV-Vis spectrophotometer (Fig. 5). Based on the results observed by the eye, the samples have been divided into three categories: evident precipitation, aggregation and colloidal suspension.

Based on eye observation and UV-Vis spectra performance, the outcomes are summarized in Tables 1 and 2 after comparing the results with those reported using other serological methods, obtained through biopsy and histology.

Out of the 30 clinical serum samples tested, 19 samples showed evident precipitation within 15 minutes and formed a pellet indicating they were positive for CD. Seven samples showed the formation of aggregated particles (without clear pellet) within 15 minutes, and those were observed for another 15 minutes for further confirmation as CD positive. In four samples, there was no precipitation or formation of aggregates

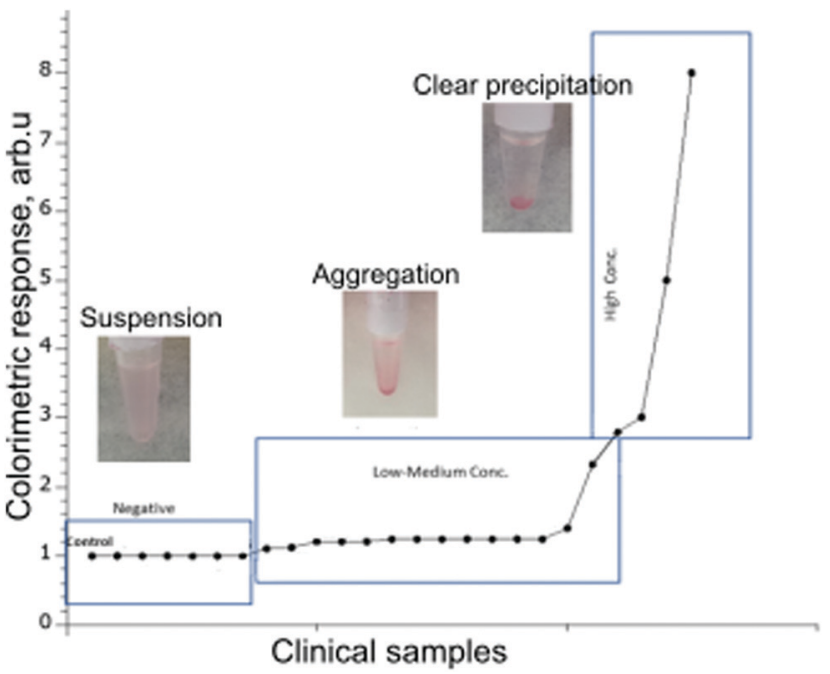

Fig. 5 Testing gliadin-coated AuNPs with patients serum samples: colorimetric response curve of different clinical samples as a result of AGA titre concentrations. Inset images: Representative images of tubes after incubation with gliadin-coated AuNPs and serum. CD negative samples presented a pink colloidal suspension, low and medium concentration samples formed a visible aggregation, and the samples with high AGA titre showed clear precipitation at the bottom of the tube.

Table 1 Test results of the patient serum samples analyzed using the precipitation AuNPs-AGA assay

\begin{tabular}{llll}
\hline & \multicolumn{2}{l}{ Disease status } & \\
\cline { 2 - 3 } & CD present & CD absent & Total \\
\hline Positive & 25 (true positive) & $1^{a}$ (false positive) & 26 \\
Negative & 4 (true negative) & 0 (false negative) & 4 \\
Total & 29 & 1 & 30
\end{tabular}

${ }^{a}$ Analysis of the samples using the AuNPs-AGA test as compared with previously existing serology.

Table 2 Test results of the patient saliva samples analyzed using the precipitation AuNPs-AGA assay

\begin{tabular}{llll}
\hline & Disease status & \\
\cline { 2 - 3 } & CD present & CD absent & Total \\
\hline Positive & 4 (true positive) & $1^{a}$ (false positive) & 5 \\
Negative & 30 (true negative) & 0 (false negative) & 25 \\
Total & 34 & 1 & 35
\end{tabular}

${ }^{a}$ Analysis of the samples using the AuNPs-AGA test as compared with previously existing serology.

within 15 minutes, and remained as a colloidal suspension, and were classified as negative for CD.

In the cohort of tested serum samples, there were two cases where the patients had previously been diagnosed with CD and therefore followed a gluten-free diet (GFD). While one person had been on a GFD for more than 8 weeks, the other person had been on a GFD for less than 2 weeks. The AuNPs-AGA based method distinguished the person following GFD for more than 
8 weeks as negative for CD, while serum from the person who has been on GFD for less than 2 weeks formed a suspension. Interestingly, the conventional serology test identified those two patients as negative, suggesting that our test can detect actual biopsy-confirmed cases even when traditional serology is negative. The analysis of these two exceptional patients using the AuNPs-AGA method illustrates that this method can potentially also be used for monitoring patients on a GFD over time. Further work using sera from more CD patients on GFD must be tested in the future to confirm further the efficacy of the test for monitoring patients on GFD.

Another encouraging result was the correct identification of an individual diagnosed with Type 1 diabetes mellitus (T1DM) as positive for CD using the AuNPs-AGA test method that essentially matched with the previously conducted biopsy and serological profile of the patient. For patients with T1DM, the prevalence of $\mathrm{CD}$ varies from $3 \%$ to $16 \%,{ }^{41}$ due to the common genetic background with multiple environmental and immunological factors. ${ }^{42}$ Also, most T1DM patients, particularly in children, present with a silent or latent form of the disease characterized by the absence of both gastrointestinal and extra-intestinal signs and are often regarded as asymptomatic. Therefore, the CD diagnosis of diabetic patients is difficult and requires continuing careful clinical and serological follow-up. ${ }^{43}$ This, however, is a single test result and needs to be further explored in more extensive clinical studies.

Three serum samples were classified as latent celiac sufferers that have positive CD serology and display mild mucosal atrophy initially, which then developed with typical atrophy of small intestine mucosa. ${ }^{41}$ In addition, four cases have been identified as "potential" CD positives; they are characterized by a normal villous architecture but demonstrate pathological findings such as increased $\gamma \delta+$ intraepithelial lymphocytes as well as the presence of gliadin specific antibodies, usually at low titres $(<1: 40) .{ }^{44}$ Although histology, in general, is considered the most reliable method of testing for $\mathrm{CD}$, it has shown lowered predictive value in recognizing "potential" CD cases. This situation requires an evaluation of serological markers for the correct diagnosis of potential CD cases, which are then monitored for the appearance of pathological symptoms.

The cohort included four samples identified as negative for CD based on biopsy. Out of the four samples, three samples were correctly identified as CD negative by AuNPs-AGA based method, while one sample showed the formation of aggregates and was identified as positive. The DGP-IgG serology titers for that sample, however, indicate positive CD necessitating a clinical follow-up on the patient to confirm CD status. As intestinal biopsy has been used as a "gold standard" for CD confirmation, that one sample has been referred to as a falsepositive result.

Overall, the results for the clinical samples tested showed that 22 of the 23 samples gave the correct result based on previous biopsy and serology. Furthermore, 7 samples were either potential or latent celiac that all tested positive for CD (Fig. S8 and S9, ESI $\dagger$ ). Finally, there was one sample that was referred to as a false positive, giving an overall accuracy for this cohort of $96.7 \%$ (Table 1 ).

In the saliva test cohort from volunteers, out of the 35 clinical saliva samples tested, four samples showed evident precipitation within 15 minutes and formed a pellet indicating they were positive for CD. The results of the 4 positive samples matched the previously conducted biopsy and serology investigations. Out of the four samples, one individual was a latent celiac sufferer and was not following a GFD when tested using the AuNPs-AGA test method. The remaining three individuals had not been following GFD strictly, and cross-contamination in the diet might have led to an increase in the titers of the AGA antibodies in the saliva.

In the other 30 samples, there was no precipitation or formation of aggregates within 15 minutes, and remained as a colloidal suspension, and were classified as negative for CD. One sample showed the formation of aggregates and identified as positive using the AuNPs-AGA test method. The intestinal biopsy, used as a "gold standard" for CD confirmation, confirmed non-CD diagnosis (but had another related autoimmune condition) and that one sample had been referred to as a false positive result in this study. Finally, the saliva analysis showed that the test method has an overall accuracy for this cohort of at least $96 \%$ (Table 2).

In future work, AGA in serum and saliva as a biomarker for CD needs further validation in larger patient cohorts, particularly in patients suffering from other auto-immune diseases. This is necessary not only to verify the diagnostic accuracy but also to confirm specificity for the diagnosis of CD.

Our developed test method offers a substantial advantage over the existing blood tests by eliminating multiple steps associated with routine serological testing, providing results within 15-30 minutes that can be determined by eye without any specialized equipment for signal reading. Finally, the developed assay is valuable for testing CD patients using saliva as a non-invasive, point of care test.

\section{Experimental}

\section{Materials and methods}

Reagents. $20 \mathrm{~nm}$ citrate stabilized gold nanoparticles (AuNPs), gliadin, bovine serum albumin (BSA), anti-gliadin antibody from rabbit, and IgG antibody from whole normal rabbit serum, Cetyl Trimethylammonium Bromide (CTAB), 4-(2-hydroxyethyl)-1-piperazineethanesulfonic acid (HEPES), Phosphate-Buffered Saline (PBS), protease inhibitor (P2714) were obtained from Sigma-Aldrich (Australia). Isopropyl alcohol was obtained from Nalgene ${ }^{\circledR}$ and ammonium sulphate was obtained from Ajax Chemicals.

Preparation of the AuNPs coated with gliadin protein. $5 \mathrm{mg}$ of gliadin was dissolved in $2.5 \mathrm{~mL}$ of aqueous CTAB $\left(1 \mathrm{mg} \mathrm{mL} \mathrm{mL}^{-1}\right)$ in a $15 \mathrm{~mL}$ FalconTM tube. The tube was then heated for 5 minutes at $60{ }^{\circ} \mathrm{C}$ and vortexed for 10 minutes. $2.5 \mathrm{~mL}$ of isopropanol was added to the tube and vortexed again for 10 minutes and sonicated for $1 \mathrm{~min}$ to completely dissolve 
gliadin. The dissolved gliadin was then filtered using a Minis$\operatorname{tart}^{\mathbb{R}}$ non-pyrogenic filter unit with a pore size of $0.2 \mu \mathrm{m}$.

$600 \mu \mathrm{L}$ of $20 \mathrm{~nm}$ AuNPs were added dropwise to the tube containing $2.6 \mathrm{~mL}$ of the filtered solubilized gliadin while vortexing. The dispersion was mixed for 60 minutes with repeated vortexing every 10 minutes, followed by centrifugation at $4500 \times g$ for 30 minutes. The supernatant was discarded, and the pellet was re-dissolved in $150 \mu \mathrm{L}$ of MilliQ water. The $20 \mathrm{~nm}$ AuNPs coated with gliadin were stored at $4{ }^{\circ} \mathrm{C}$ for up to 4 weeks.

Preparation of the AuNPs coated with BSA. $500 \mu \mathrm{L}$ of $10 \mathrm{mg} \mathrm{mL}^{-1}$ BSA dissolved in $10 \mathrm{mM}$ HEPES was added to $50 \mu \mathrm{L}$ of $20 \mathrm{~nm}$ AuNPs while vortexing. The tube was incubated for 30 minutes at room temperature with repeated vortexing. The solution was centrifuged for 30 minutes at $4500 \times g$, the supernatant discarded, and the pellet was re-suspended in $500 \mu \mathrm{L}$ MilliQ water. The process was repeated twice after which the pellet was re-suspended in $500 \mu \mathrm{L}$ HEPES buffer followed by centrifugation at $4500 \times g$ for 30 minutes using an Eppendorf ${ }^{\mathbb{R}}$ Microcentrifuge. The BSA coated AuNPs were stored at $4{ }^{\circ} \mathrm{C}$.

Characterization of protein coated AuNPs. Nanodrop (ND-1000 Nanodrop Technologies, Inc.) was performed on the AuNPs coated with gliadin and BSA to check for concentration and purity. As gliadin was dissolved in a cationic surfactant (CTAB) followed by a polar aprotic solvent (isopropanol) different controls were used to determine the background absorbance due to the use of these solvents.

The hydrodynamic diameter of nanoparticle was measured using Zetasizer Nano (Malvern Technologies, Inc.). Measurements were carried out at $25{ }^{\circ} \mathrm{C}$ in disposable cuvettes using a sample volume of $500 \mu \mathrm{L}$. Each sample was measured in duplicates, followed by mean value calculation.

High-resolution transmission electron microscopy (TEM) micrographs were obtained using a FEI Tecnai TEM $200 \mathrm{~V}$ fitted with a Gatan (Pleasantville, CA) CCD camera. Samples were prepared by placing $2 \mu \mathrm{L}$ of AuNPs adsorbed with gliadin onto a carbon-coated TEM grid (Agar Scientific, UK) and the film allowed to air dry for 15 minutes.

UV-Vis measurements were carried out using a Cary Series UV-Vis spectrophotometer (Agilent Technologies) using a standard $1 \mathrm{~cm}$ path-length quartz cuvette. Spectra were obtained from $200 \mathrm{~nm}$ to $800 \mathrm{~nm}$. MilliQ water was used as the blank.

Immunoassay for AGA in water/buffer. The immunoassay assessing the activity of AuNPs adsorbed with gliadin with the antibodies is outlined below. Assay steps were performed at room temperature. Briefly, $150 \mu \mathrm{L}$ of AuNPs adsorbed with gliadin were added to $1.5 \mathrm{~mL}$ low protein binding Eppendorf ${ }^{\mathbb{R}}$ tubes. $1 \mu \mathrm{L}$ of $20 \%$ BSA dissolved in MilliQ water was used as the blocking agent and was added to $150 \mu \mathrm{L}$ of $20 \mathrm{~nm}$ AuNPs adsorbed with gliadin. AGA $\left(1 \mathrm{mg} \mathrm{mL}{ }^{-1}\right)$ from rabbit was added to each of the tubes corresponding to concentrations ranging from $2 \mu \mathrm{g} \mathrm{mL} \mathrm{mL}^{-1}$ to $10 \mu \mathrm{g} \mathrm{mL} \mathrm{m}^{-1}$ (i.e., $1 \mu \mathrm{L}$ to $5 \mu \mathrm{L}$ ). IgG from normal rabbit serum ( $\left.1 \mathrm{mg} \mathrm{mL}^{-1}\right)$ was used as a control antibody and added to $150 \mu \mathrm{L}$ of AuNPs coated with gliadin in concentrations ranging from $2 \mu \mathrm{g} \mathrm{mL}^{-1}$ to $10 \mu \mathrm{g} \mathrm{mL}^{-1}$ $(1 \mu \mathrm{L}$ to $5 \mu \mathrm{L}$ ). MilliQ water was added to the tubes to bring the final volume in each Eppendorf tube up to $225 \mu \mathrm{L}$. The UV-
Vis absorption spectra of solutions containing gliadin coated gold nanoparticles, and AGA at increasing dilutions $\left(2 \mu \mathrm{g} \mathrm{mL}{ }^{-1}, 4 \mu \mathrm{g} \mathrm{mL}{ }^{-1}, 6 \mu \mathrm{g} \mathrm{mL}{ }^{-1}, 8 \mu \mathrm{g} \mathrm{mL}^{-1}\right.$ and $10 \mu \mathrm{g} \mathrm{mL}^{-1}$ ) were studied using UV-Vis spectrophotometer. Readings were taken in duplicate, and a student's $t$-test was used to determine the $p$-value.

Immunoassay for AGA in spiked human serum. Normal human serum was diluted to $1: 10,1: 20$ and 1:50 using $10 \mathrm{mM}$ HEPES buffer. $75 \mu \mathrm{L}$ of serum from each of the dilutions was spiked with AGA at various dilutions comparable to that seen in celiac patients.

To prevent non-specific binding, $1 \mu \mathrm{L}$ of $20 \%$ BSA dissolved in MilliQ water and was added to $150 \mu \mathrm{L}$ of $20 \mathrm{~nm}$ AuNPs adsorbed with gliadin. The tubes were incubated for 30 minutes at room temperature. $75 \mu \mathrm{L}$ of normal serum spiked with AGA at increasing dilutions of $2 \mu \mathrm{g} \mathrm{mL} L^{-1}, 4 \mu \mathrm{g} \mathrm{mL} L^{-1}, 6 \mu \mathrm{g} \mathrm{mL} L^{-1}, 8 \mu \mathrm{g} \mathrm{mL} L^{-1}$ and $10 \mu \mathrm{g} \mathrm{mL}{ }^{-1}$ was then added to $20 \mathrm{~nm}$ AuNPs adsorbed with gliadin. The tubes were then incubated for 30 minutes at room temperature.

Immunoassay for AGA in clinical human serum. Anonymized patient samples were provided by A/Prof Jason TyeDin from the Walter and Eliza Hall Institute of Medical Research (WEHI Institute, Melbourne Parkville, Australia). They were collected with informed consent and approval of Melbourne Health and WEHI Human Research Ethics Committees (2003.009 and 03/04), respectively. Ethical approval was also obtained from the University of Technology Sydney Research Ethics committee (UTS HREC ETH16-0841) before testing the clinical samples. The cohort consisted of some samples that were found to be hemolytic and had been stored for $>5$ years, and these samples were taken out to maintain assay homogeneity. In the clinical study, 30 human serum samples were analyzed in a blinded assessment. No prior knowledge of the CD status or any other clinical condition for any of the patient samples was known while testing. Samples were collected from patients with active CD (pre-treatment), treated CD (on a gluten-free diet) and controls without CD (Table 1). All cases of CD were medically diagnosed and based on typical small intestinal histology in conjunction with positive CD serology.

Before testing, each human serum sample was diluted to 1:10, 1:20 and 1:50 using $10 \mathrm{mM}$ HEPES buffer. $1 \mu \mathrm{L}$ of $20 \%$ BSA dissolved in MilliQ water was added to $20 \mathrm{~nm}$ AuNPs adsorbed with gliadin to prevent non-specific binding. $75 \mu \mathrm{L}$ of serum from each of the dilutions was then added to $20 \mathrm{~nm}$ AuNPs adsorbed with gliadin. The tubes were incubated for 15 minutes at room temperature before the absorbance was measured using a UV-Vis spectrophotometer.

Concentration of immunoglobulins in clinical human serum. $200 \mu \mathrm{L}$ of each human serum sample was centrifuged at $3200 \times g$ for 15 minutes. The supernatant was removed, and the serum samples were diluted with $200 \mu \mathrm{L}$ of $10 \mathrm{mM}$ PBS. An equal volume of saturated ammonium sulfate solution was added slowly to achieve a $33 \%$ saturated (v/v) final concentration with continuous stirring of the tubes. Samples were kept at $4{ }^{\circ} \mathrm{C}$ for 30 minutes and then centrifuged again at $2500 \times g$ for 
15 minutes. The supernatant was removed, and the pellet was re-suspended by adding $200 \mu \mathrm{L}$ of $10 \mathrm{mM}$ PBS. The concentrated serum solution was stored at $-20{ }^{\circ} \mathrm{C}$ until further use.

Zeba $^{\mathrm{TM}}$ Spin desalting columns (ThermoFisher Scientific) were used for the desalting of the immunoglobulins from the concentrated serum solution according to the manufacturer's instructions. The final concentration of the total immunoglobulins was measured using the NanoDrop and stored at $-20{ }^{\circ} \mathrm{C}$ until further use.

Immunoassay for AGA in in spiked human saliva. Human saliva samples spiked with anti-gliadin antibodies at various dilutions comparable to that seen in patients with CD were prepared. $75 \mu \mathrm{L}$ of normal human saliva was spiked with the anti-gliadin antibody at various dilutions comparable to that seen in patients with CD and was added to $150 \mu \mathrm{L} 20 \mathrm{~nm}$ AuNPs coated with gliadin, gently mixed and was allowed to incubate for 30 minutes. $1 \mu \mathrm{L}$ of $20 \%$ BSA dissolved in MilliQ water was added to $20 \mathrm{~nm}$ AuNPs adsorbed with gliadin to prevent nonspecific binding. $1 \mu \mathrm{L}$ of protease inhibitor cocktail (P2714) was added to the saliva sample to prevent protein degradation. $225 \mu \mathrm{L}$ of the sample was used for UV-Vis analysis. At least three independent experiments were conducted for each assay.

Immunoassay for AGA in clinical human saliva. Saliva sample study was conducted at the School of Life Sciences, the University of Technology Sydney, between March and December 2019. These samples were collected with the informed consent of participants and approval of the University of Technology Sydney Research Ethics committee (UTS HREC ETH18-2434) before testing the samples. In this small-scale study, 35 human saliva samples were collected from volunteers and analyzed in a blinded assessment. No prior knowledge of the CD status or any other clinical condition for any of the volunteer samples was known while testing. All cases of CD positive were confirmed with the previously conducted medical diagnosis of the volunteers and was based on typical small intestinal histology in conjunction with positive CD serology.

Prior to testing, each human saliva sample was diluted to 1:2 using $10 \mathrm{mM}$ HEPES buffer. $1 \mu \mathrm{L}$ of $20 \%$ BSA dissolved in MilliQ water was added to $20 \mathrm{~nm}$ AuNPs adsorbed with gliadin to prevent non-specific binding. $75 \mu \mathrm{L}$ of saliva from the dilution was then added to $20 \mathrm{~nm}$ AuNPs adsorbed with gliadin. $1 \mu \mathrm{L}$ of protease inhibitor cocktail (P2714) was added to the saliva sample to prevent protein degradation. The tubes were incubated for 15 minutes at room temperature before the absorbance was measured using a UV-Vis spectrophotometer.

Colorimetric response curve. A colorimetric response curve was plotted to represent the sensitivity values obtained for the different dilution values for both the AGA and the control antibody (IgG from rabbit serum). The assay sensitivity was determined based on the colorimetric response values calculated as Colorimetric Response $=I_{\max }$ at $532 \mathrm{~nm} / I$ at $550 \mathrm{~nm}$. $532 \mathrm{~nm}$ is the wavelength where AuNPs coated with gliadin shows maximum absorbance by itself (no antibodies are added) divided by the absorbance at $550 \mathrm{~nm}$, where a shift in absorbance is observed following the interaction of the antibody with the AuNP coated with gliadin.

\section{Conclusions}

In the present study, we demonstrated that gliadin-coated AuNPs could be used as an efficient tool to detect a biomarker for CD from serum and saliva. We developed a methodology to adsorb the highly hydrophobic gliadin protein on the surface of AuNPs without causing aggregation. The addition of AGA to gliadin adsorbed AuNPs at levels associated with CD resulted in color reduction and absorbance peak shift due to the aggregation of AuNPs. We confirmed that the developed assay could detect AGA not only in quantitatively spiked samples but also in a small-scale study on real CD patient's samples. The analysis of the clinical samples demonstrated not only the ease of the test procedure but also its high accuracy. This study demonstrates the immense potential of the AuNPs-AGA based assay in for pre-selecting CD sufferers for mucosal biopsy for CD as well as for monitoring the effectiveness of gluten-free diet treatment. The method can be adapted as a point-of-care test as well as in resource-limited laboratory settings for the screening to aid in early identification of CD. Future work will include more comprehensive clinical trials on a large cohort of people suffering from a variety of gastrointestinal and auto-immune conditions.

\section{Conflicts of interest}

There are no conflicts to declare.

\section{Acknowledgements}

The authors thank A/Prof Jason Tye-Din, MBBS, PhD, FRACP, Immunology Division, The Walter and Eliza Hall Institute of Medical Research, Parkville, Victoria, Australia, for providing clinical samples as well for his critical review and feedback on the manuscript. This research is supported by an Australian Government Research Training Program Scholarship. OS acknowledges the Australian Research Council, National Health and Medical Research Council (APP1101258, IH150100028) and AMP Foundation for financial support. Table of content figure and Fig. 1 were created with BioRender.com.

\section{Notes and references}

1 A. A. Kumar, J. W. Hennek, B. S. Smith, S. Kumar, P. Beattie, S. Jain, J. P. Rolland, T. P. Stossel, C. Chunda-Liyoka and G. M. Whitesides, Angew. Chem., Int. Ed., 2015, 54, 5836-5853.

2 A. Malekjahani, S. Sindhwani, A. M. Syed and W. C. W. Chan, Acc. Chem. Res., 2019, 52, 2406-2414.

3 J. F. Ludvigsson, D. A. Leffler, J. C. Bai, F. Biagi, A. Fasano, P. H. R. Green, M. Hadjivassiliou, K. Kaukinen, C. P. Kelly, J. N. Leonard, K. E. A. Lundin, J. A. Murray, D. S. Sanders, M. M. Walker, F. Zingone and C. Ciacci, Gut, 2013, 62, 43.

4 M. N. Marsh, V. Villanacci and A. Srivastava, Gastroenterol. Hepatol. Bed Bench, 2015, 8, 171-177.

5 E. Tonutti and N. Bizzaro, Autoimmun. Rev., 2014, 13, 472-476.

6 G. Oberhuber, G. Granditsch and H. Vogelsang, Eur. J. Gastroenterol. Hepatol., 1999, 11, 1185. 
7 J. A. Tye-Din, D. J. Cameron, A. J. Daveson, A. S. Day, P. Dellsperger, C. Hogan, E. D. Newnham, S. J. Shepherd, R. H. Steele, L. Wienholt and M. D. Varney, Intern. Med. J., 2015, 45, 441-450.

8 I. Dahlbom, B. I. Nyberg, L. Berntson and T. Hansson, Scand. J. Clin. Lab. Invest., 2016, 76, 208-216.

9 F. Benkebil, C. Combescure, S. I. Anghel, C. Besson Duvanel and M. G. Schäppi, World J. Gastroenterol., 2013, 19, 5111-5117.

10 A. Kaur, O. Shimoni and M. Wallach, J. Gastroenterol., 2017, 52, 1001-1012.

11 R. Banerjee and A. Jaiswal, Analyst, 2018, 143, 1970-1996.

12 B. A. Rohrman, V. Leautaud, E. Molyneux and R. R. RichardsKortum, PLoS One, 2012, 7, e45611.

13 E. I. Laderman, E. Whitworth, E. Dumaual, M. Jones, A. Hudak, W. Hogrefe, J. Carney and J. Groen, Clin. Vaccine Immunol., 2008, 15, 159-163.

14 A. L. Tomás, M. P. de Almeida, F. Cardoso, M. Pinto, E. Pereira, R. Franco and O. Matos, Front. Microbiol., 2019, 10, 2917, DOI: 10.3389/fmicb.2019.02917.

15 J. Su, Z. Zhou, H. Li and S. Liu, Anal. Methods, 2014, 6, 450-455.

16 R. E. Biagini, D. L. Sammons, J. P. Smith, B. A. MacKenzie, C. A. F. Striley, J. E. Snawder, S. A. Robertson and C. P. Quinn, Clin. Vaccine Immunol., 2006, 13, 541-546.

17 N. Tippkötter, H. Stückmann, S. Kroll, G. Winkelmann, U. Noack, T. Scheper and R. Ulber, Anal. Bioanal. Chem., 2009, 394, 863-869.

18 Q. Li, L. Liu, W. Chen, C. Peng, L. Wang and C. Xu, Int. J. Environ. Anal. Chem., 2009, 89, 261-268.

19 S. Gupta, H. Andresen, J. E. Ghadiali and M. M. Stevens, Small, 2010, 6, 1509-1513.

20 R. Chandrawati and M. M. Stevens, Chem. Commun., 2014, 50, 5431-5434.

21 F. Bienvenu, C. Besson Duvanel, C. Seignovert, P. Rouzaire, A. Lachaux and J. Bienvenu, Eur. J. Gastroenterol. Hepatol., 2012, 24, 1418-1423.

22 V. Abadie, L. M. Sollid, L. B. Barreiro and B. Jabri, Annu. Rev. Immunol., 2011, 29, 493-525.

23 G. Malamut, R. El Machhour, N. Montcuquet, S. MartinLannerée, I. Dusanter-Fourt, V. Verkarre, J. J. Mention, G. Rahmi, H. Kiyono, E. A. Butz, N. Brousse, C. Cellier, N. Cerf-Bensussan and B. Meresse, J. Clin. Invest., 2010, 120, 2131-2143.
24 R. Elghanian, J. J. Storhoff, R. C. Mucic, R. L. Letsinger and C. A. Mirkin, Science, 1997, 277, 1078.

25 J.-S. Lee, M. S. Han and C. A. Mirkin, Angew. Chem., Int. Ed., 2007, 46, 4093-4096.

26 Q. Wang, R. Liu, X. Yang, K. Wang, J. Zhu, L. He and Q. Li, Sens. Actuators, B, 2016, 223, 613-620.

27 J. M. Slocik, A. O. Govorov and R. R. Naik, Nano Lett., 2011, 11, 701-705.

28 R. A. Sperling and W. J. Parak, Philos. Trans. R. Soc., A, 2010, 368, 1333-1383.

29 C.-C. Huang, Y.-F. Huang, Z. Cao, W. Tan and H.-T. Chang, Anal. Chem., 2005, 77, 5735-5741.

30 S. H. Brewer, W. R. Glomm, M. C. Johnson, M. K. Knag and S. Franzen, Langmuir, 2005, 21, 9303-9307.

31 J. C. Watson and W. F. Thompson, Methods in Enzymology, Academic Press, 1986, pp. 57-75, vol. 118.

32 Y. Li, R. Xin, D. Zhang and S. Li, Crop J., 2014, 2, 10-21.

33 D. Tsai, F. W. DelRio, A. M. Keene, K. M. Tyner, R. I. MacCuspie, T. J. Cho, M. R. Zachariah and V. A. Hackley, Langmuir, 2011, 27, 2464-2477.

34 S. Link and M. A. El-Sayed, J. Phys. Chem. B, 1999, 103, 4212-4217.

35 H. Jans, X. Liu, L. Austin, G. Maes and Q. Huo, Anal. Chem., 2009, 81, 9425-9432.

36 L. Jürgens, A. Nichtl and U. Werner, Cytometry, 1999, 37, 87-92.

37 C. O'Farrelly, J. Kelly, W. Hekkens, B. Bradley, A. Thompson, C. Feighery and D. Weir, Br. Med. J., 1983, 286, 2007-2010.

38 H. F. al-Bayaty, M. J. Aldred, D. M. Walker, R. G. Newcombe, G. Swift, P. M. Smith and P. J. Ciclitira, J. Oral Pathol. Med., 1989, 18, 578-581.

39 M. Lenander-Lumikari, R. Ihalin and H. Lähteenoja, Arch. Oral Biol., 2000, 45, 347-354.

40 N. T. Tucker, F. S. Barghuthy, T. J. Prihoda, V. Kumar, A. Lerner and E. Lebenthal, J. Pediatr., 1988, 113, 286-289.

41 U. Volta, F. Tovoli and G. Caio, Expert Rev. Gastroenterol. Hepatol., 2011, 5, 479-487.

42 G. K. Holmes, Diabetic Med., 2001, 18, 169-177.

43 K. Kaukinen, M. Mäki, J. Partanen, H. Sievänen and P. Collin, Dig. Dis. Sci., 2001, 46, 879-887.

44 U. Volta and V. Villanacci, Cell. Mol. Immunol., 2011, 8, 96-102. 